\title{
EEG Signal Analysis for Identification of Epilepsy Using Machine Learning Classification Approaches
}

\author{
Sk. Ebraheem Khaleelulla, Dr. P. Rajesh Kumar
}

\begin{abstract}
Epilepsy is censorious neurological disorder in which nerve cell activity in the brain is disturbed causing recurrent seizures which are sudden, uncontrolled electrical discharges in the brain cell. In clinical treatment of epileptic patients seizure reorganization has much prominence. Hence in detecting the phenomenon of epilepsy Electroencephalogram (EEG) signal is widely used as it includes important carnal data of the brain. Though it is critical to analyze the EEG signal and identify the seizures. So feature extraction of EEG signal plays a vital role for epilepsy detection. This paper describes an worthwhile feature extraction based on variational mode decomposition (VMD) to identify epilepsy. The extracted features fed to ANN, KNN and $S V M$ in order to classify epilepsy. The performance of the SVM classifier shows the better classification compared to existing methods.
\end{abstract}

Index Terms: Electroencephalogram, Variational Mode Decomposition, Artificial Neural Network, K-Nearest Neighbor, Support Vector Machine.

\section{INTRODUCTION}

Epilepsy is the most critical neurological disorder which originates from the brain electrical activity having abnormal discharges called seizures leading to uncontrollable movements [1]. Epilepsy is widespread neurological disease affecting about $1 \%$ of the world's population. Epilepsy is very hard to diagnose In spite of its spread and can be challenging in detecting the disorder [2]. People with epilepsy are more prone to die too early compared with others. Hence, predicting and diagnosing the epileptic seizures plays important role for better prevention and cure for patients. Clinically EEG signals are used to observe the brain electrical activities which contain the markers of epilepsy. Through the analysis of EEG signal we can predict and diagnose epileptic seizures. Electroencephalogram (EEG) is a test that detects abnormalities in the brain waves, or in the electrical activity of the brain. The procedure involves the electrodes of small metal discs which are pasted on the scalp of patient. These electrodes detect tiny electrical charges that result from the activity of brain cells. EEG is an electrophysiological monitoring method and contains much worthy information which relates to physiological activity of the brain and thus is a very important tool in analyzing and understanding various neurological disorders like epilepsy [3].
Two states of abnormal activities are shown in epileptic patient's in their EEG signals namely seizure free or interictal and ictal [4]. The characteristic of an epilepsy disorder which refers to the period between seizures and exhibit spikes or sharp waves in interictal EEG signals. The physiologic state or event such as seizure or stroke with perpetual waveforms refers to the ictal EEG signals. Epilepsy can be exposed to view the long durations of EEG signals by conventional methods with the help of experienced neurophysiologists by their visual inspection, perhaps it is individual and tedious. Epileptic EEG signals with computer-aided detection (CAD) can be used in order to vanquish these obstacles.

The frequency range of electrical waves of the brain is from $4 \mathrm{~Hz}$ to $80 \mathrm{~Hz}$ and small amplitude (approximately $100 \mu \mathrm{V}$ ). Each EEG signal is normally divided into five sub-bands: Delta $(0.5 \mathrm{~Hz}-4 \mathrm{~Hz})$, Theta $(4 \mathrm{~Hz}-8 \mathrm{~Hz})$, Alpha $(8 \mathrm{~Hz}-12$ $\mathrm{Hz})$, Beta $(13 \mathrm{~Hz}-30 \mathrm{~Hz})$, and Gamma $(30 \mathrm{~Hz}-60 \mathrm{~Hz})[5,6]$. The behavior of EEG signals can be best depicted by extracting the features and these are prominent for performance of seizure detection. EEG signals hidden distinctive characteristics are captured with feature extraction and appropriate optimization of the features influence the final classification accuracy.

The frequency based Frequencies and energies of the EEG signals in different sub-band features are used with ANN to classify the epileptic seizure with normal EEG [7]. The time based mean and spectral based bispectral entropy, squared entropy and phase entropy features input to KNN to classify the epileptic seizure [8]. The time based features mean, maximum value, minimum value, standard deviation is used with ANN to classify the epileptic seizure to the normal EEG [9]. The time based mean, absolute, median, variance features input to feed-forward neural network to classify epileptic seizure to the normal EEG [10]. The time based mean, standard deviation and frequency based FFT and wavelet transform features are used with SVM to classify epileptic seizure with normal EEG [11].

The time based entropy, approximation entropy, hurust exponent features are input to SVM to classify epileptic seizure with normal EEG [12]. Time based mean, median, maximum, minimum, range, standard deviation, absolute deviation features are used with ANN, SVM, and KNN for epileptic seizure with normal EEG classification [13]. 
Several methods are proposed and developed based on the scheme: EEG data of feature extraction in frequency domain [13], time domain [14], and time-frequency domain [12][15][16]. The review of EEG features has been propounded for classifying and detecting the epileptic seizure.

The EEG dataset is adopted from the Bonn University Hospital of Freiburg [17]. It contains five individual subsets (set A-E) named as Z,O,N,F and S. Each subset consists 23.6s duration of 100 single channel EEG signals. The data has a sampling rate of $173.61 \mathrm{~Hz}$ and digitalized with 12 bit analog to digital resolution. Set A and set B are captured extra cranially whereas remaining collected intracranial with standardized 10-20 electrode system. Set A was recorded from the healthy patients when eyes open, set B was recorded from the healthy patients when eyes close, Set C and set D were recorded when patients are in seizure free intervals, Set E consists of epileptic seizure signals and these signals show ictal activity.

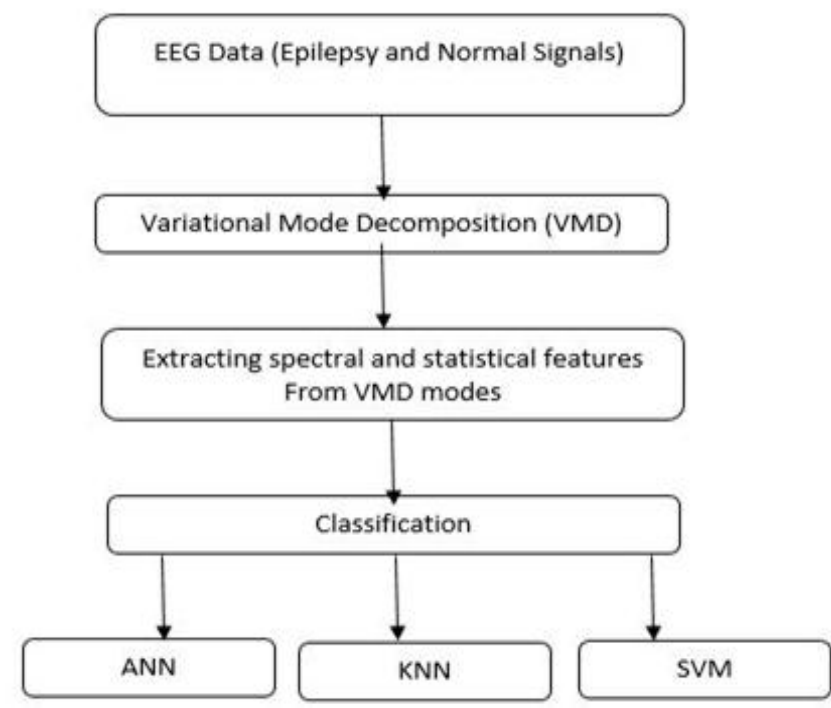

Fig. 1: EEG signals classification Overview diagram using (variational mode decomposition)VMD method.

The reminder of this paper is organised as fallows. In Section-II, VMD methodology, feature extraction in VMD domain and three classifiaction techniques are introduced. With the extracted features performaces of each classifier with respect their confusion plots for finding performance perameters discussed in section-III. Comparison between some of the researches and experimental results discussed in the same section. Finally concludes in Section-IV

\section{VARIATIONAL MODE DECOMPOSITION}

Variational mode decomposition (VMD) is a new adaptive signal decomposition, It decomposes any real time signal into a band limited functions or variational modes $\left(\mathrm{u}_{\mathrm{k}}\right)$. Each mode occurred concurrently and exhibits the sparsity property for reconstruction of an input signal. VMD decomposes a real time signal into $\mathrm{k}$ modes $\left(\mathrm{u}_{\mathrm{k}}\right)$ around its center frequency $(\omega)$. Hilbert transform and frequency shifting property are useful parameters in formulation of an optimization problem. The formulation of a constrained variational problem as [24],

$$
\begin{gathered}
\min _{\left\{u_{k}\right\},\left\{\omega_{k}\right\}}\left\{\sum_{k}\left\|\partial_{t}\left[\left(\delta(t)+\frac{j}{\pi t}\right) * u_{k}(t)\right] e^{-j \omega_{k} t}\right\|_{2}^{2}\right\} \\
\sum_{k} u_{k}=f
\end{gathered}
$$

The quadratic penalty factor and Lagrangian multiplier $(\lambda)$ are converts into (2) from an (1), The unconstrained optimization problem is expressed as (2)

$$
\begin{aligned}
& \ell\left(\left\{u_{k}\right\},\left\{\omega_{k}\right\}, \lambda\right):=\alpha \sum_{k}\left\|\partial_{t}\left[\left(\delta(t)+\frac{j}{\pi t}\right) * u_{k}(t)\right] e^{-j \omega_{k} t}\right\|_{2}^{2}+ \\
& \left\|f(t)-\sum_{k} u_{k}(t)\right\|_{2}^{2}+\left\langle\lambda(t), f(t)-\sum_{k} u_{k}(t)\right\rangle
\end{aligned}
$$

The alternate direction method of a multiplier (ADMM) is an optimization method to solve (2) lagrangian function $\ell$, it estimate modes around its own center frequencies. The wiener filter is embedded in a VMD to update each mode $u_{k}(\omega)$ optimally in a spectral domain.

Algorithm1: Decomposition of EEG signals by using VMD algorithm

Step-1: predefine $K$ which is the number of modes.

Step-2: Initializing of $\left\{\hat{u}_{k}^{1}\right\},\left\{\omega_{k}^{1}\right\}, \hat{\lambda}^{1}$, and $n=0$;

Step-3: For $n=n+1$, repeat loop until $k=1: K$ for $\omega \geq 0$. It keep on change $\hat{u}_{k}(t)$ in the spectral domain is [24],

$$
\begin{aligned}
& \hat{u}_{k}^{n+1}(\omega) \leftarrow \frac{\hat{f}(\omega)-\sum_{i<k} \hat{u}_{k}^{n+1}(\omega)-\sum_{i>k} \hat{u}_{k}^{n}(\omega)+\frac{\hat{\lambda}^{n}(\omega)}{2}}{1+2 \alpha\left(\omega-\omega_{k}^{n}\right)^{2}} \\
& \hat{u}_{k}^{n+1}(t)=\operatorname{Real}\left\{\operatorname{ifft}\left(\hat{u}_{k}^{n+1}(\omega)\right)\right\}
\end{aligned}
$$

Update $\omega_{k}$ with

$$
\omega_{k}^{n+1} \leftarrow \frac{\int_{0}^{\infty} \omega\left|\hat{u}_{k}^{n+1}(\omega)\right|^{2} d \omega}{\int_{0}^{\infty}\left|\hat{u}_{k}^{n+1}(\omega)\right|^{2} d \omega}
$$

Step-4: Assign $k=k+1$, repeat until $\mathrm{k}$ equals $\mathrm{K}$ and $\mathrm{n}$ iteration of the loop

Step-5: $\lambda$ ( Lagrange multiplier), updated for all $\omega \geq 0$ based on dual-ascent

$$
\hat{\lambda}^{n+1}(\omega) \leftarrow \hat{\lambda}^{n}(\omega)+\tau\left(\hat{f}(\omega)-\sum_{k} \hat{u}_{k}^{n+1}(\omega)\right)
$$

Step-6: Repeat the above steps 2 to 5 until to obtain the modes by satisfy the convergence condition.

$$
\sum_{k=1}^{K} \frac{\left\|\hat{u}_{k}^{n+1}-\hat{u}_{k}^{n}\right\|_{2}^{2}}{\left\|\hat{u}_{k}^{n}\right\|_{2}^{2}}<\varepsilon
$$


Here $\in, \wedge$ and $\tau$ represents the tolerance Fourier transform and time steps of dual ascent convergence respectively. Ifft ( ), Real ( ) are represents the inverse Fourier transform and real part of analytic signal. In VMD, the selection of a parameter is a first task, a number of modes $(K)$, and alpha.

The mode decomposition of normal and epilepsy EEG signals are in Figure 2.2a, b shown respectively. It signifies that the mode increases also its frequency increases.
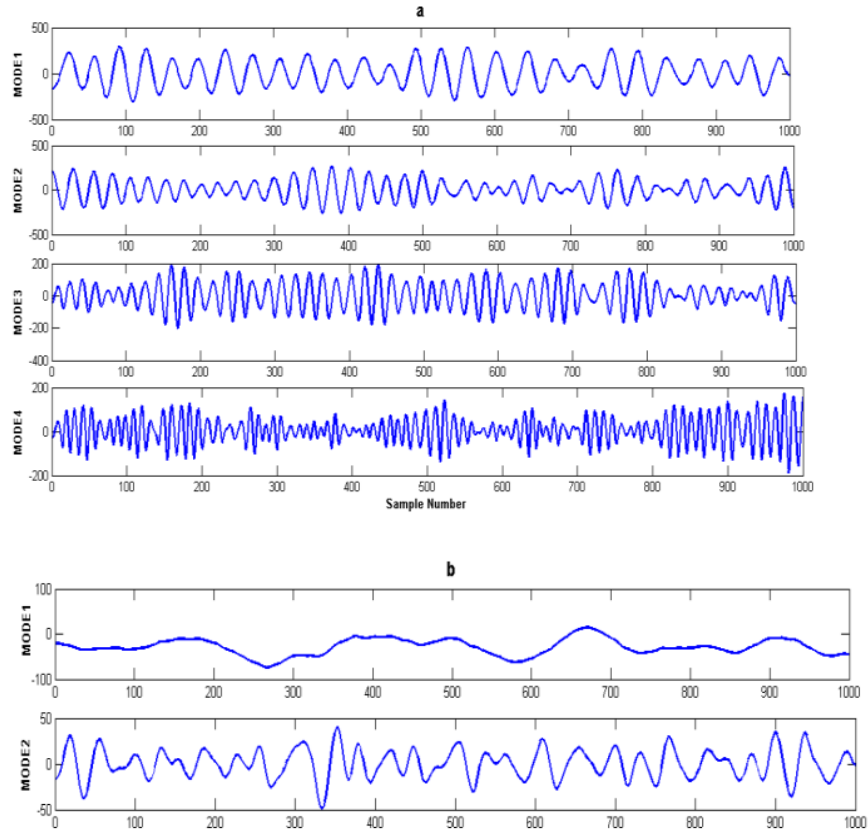

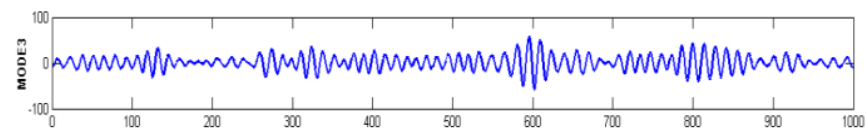
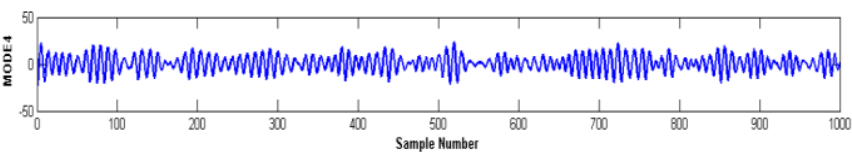

Figure 2: VMD of four modes (a) Epilepsy signal (b) Normal EEG signal

A. Feature extraction: examples.

\section{1) Statistical features:}

For an $\mathrm{N}$ sample EEG signal $y[n]$ gives the magnitude spectrum of $Y[m]$.

Statistical features:

Mean: It is the average of an N sample EEG signal; it can be defined [21],

$$
\mu=\frac{1}{N} \sum_{i=1}^{N} Y_{i}
$$

Standard deviation: The dispersion of data from it's a mean value of a signal is a standard deviation. It is derived as [20],

$$
\text { EG signal; it } \sqrt{\frac{\mathrm{anl}}{N-1} \sum_{i=1}^{N}\left(Y_{i}-\mu\right)^{2}}
$$

Coefficient of variation ( $\mathrm{COV})$ : The ratio of standard deviation to mean value of the EEG signal is a coefficient of variation. It can be expressed as [19].

$$
\operatorname{COV}=\frac{\sigma}{\mu}
$$

Entropy (H): It is defined as the measuring of randomness in EEG signals. For an EEG signals with $\mathrm{N}$ number of samples ( $\left.y_{1}, \mathrm{y}_{2}, \mathrm{y}_{3}, \ldots . Y_{N}\right)$ is expressed as [19],

$$
H(Y)=-\sum_{i=1}^{N} p\left(y_{i}\right) \log \left(p\left(y_{i}\right)\right) \quad p\left(y_{i}\right)=\left[p\left(y_{1}\right), p\left(y_{2}\right) \ldots . . .\right]
$$

Inter quartile range (IQR): It is defined as the difference between $75^{\text {th }}$ and $25^{\text {th }}$ percentile of samples, It measures variability in a data set is given by [18],

$$
I Q R=\mathrm{Q}_{3}-\mathrm{Q}_{1}
$$

Here, Q3 and Q1 are third and first quartile respectively.

Skewness: It measures the symmetry shape of the distribution of a signal, It can be derived as

$$
\text { Skewness }=\frac{1}{N} \sum_{i=1}^{N}\left(\frac{y_{i}-\mu}{\sigma}\right)^{3}
$$

NegEntropy: The differences between gaussian entropy $\mathrm{H}$ (Ygauss) and differential entropy $\mathrm{H}(\mathrm{Y})$ having mean $\mu$ and variance $\sigma^{2}$ of EEG signals. It is expressed as [20],

$$
J(Y)=H\left(Y_{\text {gauss }}\right)-H(Y) \quad H\left(Y_{\text {gauss }}\right)=\frac{1}{2} \log \left(2 \pi e \sigma^{2}\right)
$$

Kurtosis: It is a one of the statistical moment; it gives the time series data peaked nature. Kurtosis can be derived as [25]

$$
k=\frac{1}{N} \sum_{i=1}^{N}\left(\frac{y_{i}-\mu}{\sigma}\right)^{4}
$$

\section{2) Spectral features:}

Spectral flatness (SF): Spectral flatness can be define as the ratio of magnitude spectrum of geometric mean to the arithmetic mean, It can be expressed as [23,24],

$$
S F=\frac{\prod_{m=0}^{N-1}|Y[m]|^{\frac{1}{N}}}{\frac{1}{N} \sum_{m=0}^{N-1}|Y[m]|}
$$

Spectral spread (SS): Average deviation of a magnitude spectrum around its spectral centroid is called as spectral spread. It can also be assumed as instantaneous bandwidth, It can be mathematically expressed as $[23,24]$,

$$
S S=\frac{\sum_{m=0}^{N-1}(m-S C)^{2}|Y[m]|}{\sum_{m=0}^{N-1}|Y[m]|}
$$

It is a ratio of sum of a weighted magnitude spectrum to normalized by an unweighted sum is called as spectral centroid (SC),

$$
S C=\frac{\sum_{m=0}^{N-1} m|Y[m]|}{\sum_{m=0}^{N-1}|Y[m]|}
$$

Spectral decrease (SDec): It measuring the amount of decrease of a spectral envelope of a signal with respect to frequency, it is denoted as[24], 


$$
\operatorname{SDec}=\frac{\sum_{m=1}^{N-1} \frac{1}{m} \cdot(|Y[m]|-|Y[0]|)}{\sum_{m=1}^{N-1}|Y[m]|}
$$

K-Nearest Neighbor Classifier (KNN): KNN is one of the finest learning algorithm, Until the classification whole calculation differs in which function approximated locally only. KNN algorithm is one of the simplest Machine Learning algorithms. In order to catalogue the unknown instance with class label as that of known neighbor and to locate the nearest neighbor in instance set. Classification with an instant classifier can be straight forward task. Class membership is the output in KNN classification to classify, the training and testing datasets of epileptic EEG classification are applied to $\mathrm{KNN}$ and the documentation of classification result is done.

Artificial Neural Network (ANN): In machine learning and cognitive science a family of statistical learning models are said to be artificial neural networks and are inspired by biological neural networks. Using machine learning process, the training dataset is used to train ANN, for epileptic seizure classification using ANN the test EEG dataset is used for testing the performance.

Support Vector Machines (SVM): For Classification of healthy EEG signal, SVM classifier is fed with above features. In two-class problem, decision function is given by

$$
g(x)=\operatorname{sign}\left[w^{T} f(x)+b\right]
$$

Optimization problem can be expressed as

$$
\begin{array}{r}
\text { Minimize } \quad J(w, b, e)=\frac{1}{2} w^{T} w+\frac{\gamma}{2} \sum_{i=1}^{N} e_{i}^{2} \\
\text { Subject to } \quad y_{i}\left[w^{T} f\left(x_{i}\right)+b\right]=1-e_{i}, \quad i=1,2 \ldots, N
\end{array}
$$

Here $x_{i}$ is $\mathrm{N}$ input with $i^{\text {th }}$ feature vectors and $y_{\mathrm{i}}$ is class label of 1 or -1 for $x_{i}$. b is called bias term and $\gamma$ is called parameter of regularization. $\alpha_{i}$ is known as lagrangian multiplier, its SVM classifier solution is obtained as

$$
g(x)=\operatorname{sign}\left[\sum_{i=1}^{N} \alpha_{i} y_{i} K\left(x, x_{i}\right)+b\right]
$$

Kernel is needed for SVM classifier for training. In this Gaussian RBF kernel is the efficient one. RBF kernel can be represented as

$$
K\left(x, x_{i}\right)=f^{T}(x) f\left(x_{i}\right)=e^{\left(-\left\|x-x_{i}\right\|^{2} / 2 \sigma^{2}\right)}
$$

Parameter $\sigma$ is an optimization kernel width. The SVM classifier detailed information is available in [25, 9].

\section{RESULTS AND DISCUSSION}

In this paper we proposed, designed, developed and implemented an efficient approach for classification of the epileptic and normal EEG with the aid of VMD based features. The proposed epileptic seizure classification algorithms are implemented and simulated in the Matlab environment and the simulation results are presented in this section. Simulation results of an epileptic seizure classification algorithm with ANN are presented as follows in Table 1.

According to the confusion matrix obtained, it is observed that, the classification algorithm using ANN achieves a overall accuracy of $85 \%$, overall sensitivity of $82.50 \%$, overall specificity of $87.50 \%$, overall precision of $86.84 \%$, overall f_measure of 84.614 and overall gmean $84.96 \%$.

Table 1: Confusion matrix of Epileptic Seizure Classification using

\begin{tabular}{lll}
\multicolumn{3}{c}{ ANN } \\
\hline Signal & Epilipsy(\%) & Normal(\%) \\
\hline Epilipsy & 86.8 & 13.2 \\
Normal & 16.7 & 83.3 \\
\hline
\end{tabular}

Simulation results of an epileptic seizure classification algorithm with KNN are presented as follows in Table 2. According to the confusion matrix obtained, it is observed that, the classification algorithm using KNN achieves a overall accuracy of $88.75 \%$, overall sensitivity of $94.17 \%$, overall specificity of $83.33 \%$, overall precision of $84.96 \%$, overall f_measure of 89.33 and overall gmean $88.58 \%$.

Table 2: Confusion matrix of Epileptic Classification using KNN

\begin{tabular}{lll}
\hline \multicolumn{1}{c}{ Signal } & Epilipsy(\%) & Normal $(\%)$ \\
\hline Epilipsy & 85.0 & 15.0 \\
Normal & 6.5 & 93.5
\end{tabular}

Finally the simulation results of an SVM based epileptic seizure classification algorithm are illustrated in Table 3. From the confusion matrix of SVM based epileptic seizure classification algorithm, it is observed that SVM approach achieves all time high classification performance compared to ANN and KNN. It achieves a overall accuracy of $92.08 \%$, overall sensitivity of $86.33 \%$, overall specificity of $100 \%$, overall precision of $100 \%$, overall f_measure of 92.66 and overall gmean $92.91 \%$.

Table 3: Confusion matrix of Epileptic Classification using SVM

\begin{tabular}{rll}
\hline Signal & Epilipsy(\%) & Normal(\%) \\
\hline Epilipsy & 86.3 & 13.7 \\
Normal & 0.0 & 100 \\
\hline
\end{tabular}

Table 4: Performance summary of an epileptic seizure classification

\begin{tabular}{lllll}
\hline S.No & Parameter & ANN & KNN & \multicolumn{1}{c}{ SVM } \\
\hline 1 & Accuracy & 85.00 & 88.75 & 92.08 \\
2 & Sensitivity & 82.50 & 94.17 & 86.33 \\
3 & Specificity & 87.50 & 83.33 & 100 \\
4 & Precision & 86.84 & 84.96 & 100 \\
5 & f_measure & 84.64 & 89.33 & 92.66 \\
6 & gmean & 84.96 & 88.58 & 92.91 \\
\hline
\end{tabular}

From the comparison, it is observed that SVM classifier based epileptic seizure classification algorithm show predominantly better classification accuracy over the classification algorithms based on ANN and KNN. All the performance parameters of SVM shown better results compared to ANN and KNN. 
As evident from Table II, proposed method compared to the work from literature achieved high accuracy rate.
Furthermore, new features and decomposing technique in the proposed scheme is a substantial improvement.

Table 5: Comparison table

\begin{tabular}{|c|c|c|c|c|}
\hline Title & Author & Features & Classifier & Results \\
\hline $\begin{array}{l}\text { Neural Network Classifier for the } \\
\text { Detection of Epilepsy (2013) }\end{array}$ & $\begin{array}{l}\text { V.Udayashankara } \\
\text { G.R.Kiranmayi } \\
\text { [12] }\end{array}$ & $\begin{array}{l}- \text { non- redundant region } \\
\text { maximum value } \\
\text {-non- redundant region } \\
\text { minimum value } \\
\text {-non-redundant region mean } \\
\text { value, principal diagonal } \\
\text { maximum value } \\
\text {-principal diagonal minimum } \\
\text { value }\end{array}$ & $\begin{array}{l}\text { Artificial } \\
\text { neural } \\
\text { networks } \\
\text { (ANN) }\end{array}$ & $81.67 \%$ \\
\hline $\begin{array}{l}\text { EEG based detection of Epilepsy by a } \\
\text { Mixed Design Approach (2016) }\end{array}$ & $\begin{array}{l}\text { DawoodDilberJasleen } \\
\text { Kaur [27] }\end{array}$ & $\begin{array}{l}\text {-mean } \\
\text {-(SD)Standard deviation } \\
\text {-variance } \\
\text {-(FFT) fast fourier transform } \\
\text {-(WT) wavelet transform }\end{array}$ & $\begin{array}{l}\text { Support } \\
\text { Vector } \\
\text { Machine }\end{array}$ & $70 \%$ \\
\hline $\begin{array}{l}\text { Epileptic Seizure Detection Using Non } \\
\text { Linear Analysis of EEG (2016) }\end{array}$ & vijith V S et al. [26] & $\begin{array}{l}\text {-AproximateEntropy } \\
\text { - Sample Entropy } \\
\text { - Hurst exponent }\end{array}$ & $\begin{array}{l}\text { Support } \\
\text { Vector } \\
\text { Machine }\end{array}$ & $\begin{array}{l}89 \% \\
91 \%\end{array}$ \\
\hline $\begin{array}{l}\text { Epileptic Seizure Classification using } \\
\text { Statistical Features of EEG Signal } \\
\text { (2017) }\end{array}$ & $\begin{array}{l}\text { Mohiuddin Ahmad } \\
\text { Md.Mamun Rashid } \\
\text { [28] }\end{array}$ & $\begin{array}{l}\text {-median } \\
\text {-mean } \\
\text {-max norm } \\
\text {-minimum } \\
\text {-range } \\
\text {-mean absolute deviation } \\
\text {-median absolute } \\
\text { deviation } \\
\text {-maximum } \\
\text {-(SD) standard deviation } \\
\text {-12 norm }\end{array}$ & $\begin{array}{l}\text { Neural } \\
\text { Network (NN) }\end{array}$ & $78.7 \%$ \\
\hline Proposed Method & Sk Ebraheem Khaleelulla & $\begin{array}{l}\text {-Spectral Spread } \\
\text {-Spectral Flatness } \\
\text {-spectral decrese and some } \\
\text { stastical features }\end{array}$ & $\begin{array}{l}\text { ANN } \\
\text { KNN } \\
\text { SVM }\end{array}$ & $\begin{array}{l}85 \% \\
88.75 \% \\
92.08 \% \\
\end{array}$ \\
\hline
\end{tabular}

\section{CONCLUSION} variable mode decomposition on various epilepsy and normal signals to extract the statistical and spectral features. An effective feature extraction method for EEG is developed by decomposing the signal into four level for both training and testing sets and totally 44 features are calculated. Demonstrated the way to incorporate the identification of epileptic seizure by various algorithms such as ANN, KNN, SVM. The accuracy, sensitivity, specificity, precision, f_mesure, gmean is observed for each algorithm. Out of this SVM achieves high classification accuracy compared to ANN and KNN. Hence, SVM classifier is better in identification on epileptic seizure.

\section{REFERENCES}

1. Dehuri, Satchidanada, Alok Kumar Jagadev, and Sung-Bae Cho "Epileptic Seizure Identification from Electroencephalography Signal Using DE-RBFNs Ensemble." Procedia Computer Science23 (2013): 84-95.

2. Josephson, Colin B., Sherry Sandy, Nathalie Jette, Tolulope T. Sajobi, Deborah Marshall, and Samuel Wiebe. "A Systematic Review of Clinical Decision Rules for Epilepsy." Epilepsy \& Behavior 57 (2016): 69-76.

3. Guo, Ling, Daniel Rivero, Julián Dorado, Cristian R. Munteanu, and Alejandro Pazos. "Automatic Feature Extraction Using Genetic
In this paper, the proposed approach initially performs

Programming: An Application to Epileptic EEG Classification." Expert Systems with Applications 38, no. 8 (2011): 10425-0436.

4. Acharya, U. Rajendra, H. Fujita, Vidya K. Sudarshan, Shreya Bhat, and Joel E.w. Koh. "Application of Entropies for Automated Diagnosis of Epilepsy Using EEG Signals: A Review." Knowledge-Based Systems 88 (2015): 85-96.

5. Ebraheem Khaleelulla S., Rajesh Kumar P.” EEG Signal Analysis for Mental States and Conditions of Human Brain”. In: Satapathy S., Bhateja V., Chowdary P., Chakravarthy V., Anguera J. (eds) Proceedings of 2nd International Conference on Micro-Electronics, Electromagnetics and Telecommunications. Lecture Notes in Electrical Engineering, vol 434. (2018) Springer, Singapore

6. Kalaivani, M., V. Kalaivani, and V. Anusuya Devi. "Analysis of EEG Signal for the Detection of Brain Abnorrnalities." JJCA Proceedings on International Conference on Simulations in Computing Nexus. No. 2. Foundation of Computer Science (FCS), 2014.

7. R.S. Fisher, W.E. Boas, W. Blume, C. Elger, P. Genton, P. Lee, and J. Engel, "Epileptic seizures and epilepsy:" Definitions proposed by the International League Against Epilepsy (ILAE) and the International Bureau for Epilepsy (IBE), Epilepsia, vol. 46(4), 2005, pp. 470-472.

8. R.S. Fisher, C. Acevedo, A. Arzimanoglou, A. Bogacz, J.H. Cross, C.E.Elger, J. Engel, L. Forsgren, J.A. French, M. Glynn, D.C. Hesdorffer,B.I. Lee, G.W. Mathern, S.L. Mosh’e, E. Perucca, I.E Scheffer, T.Tomson, M. Watanabe, and S. Wiebe, ILAE official report: "A practical clinical definition of epilepsy. ”Epilepsia, vol. 55(4), 2014, pp. 475-482.

9. B. Abou Khalil and K. E. Misulis, Atlas of EEG \& Seizure Semiology, Elsevier Ed., 2006. 
10. H. R. Mohseni, A. Maghsoudi, and M. B. Shamsollahi, "Seizure detection in EEG signals: A comparison of different approaches", International Conference of IEEE Engineering in Medicine and Biology Society (EMBS), 2006, pp. 6724-6727.

11. R. G. Andrzejak et al., "Indications of nonlinear deterministic and finite-dimensional structures in time series of brain electrical activity: dependence on recording region and brain state," Phys. Rev. E, vol. 64, no. 6, Nov. 2001, pp. 116-126.

12. G. Kiranmayi and V. Udayashankara, "Neural network classifier for the detection of epilepsy," in Circuits, Controls and ommunications (CCUBE), 2013 International conference on 2013, pp. 1-4.

13. L. Boubchir, S. Al-Maadeed, A. Bouridane, and A . Ali Cherif,"Time frequency image descriptors-based features for EEG epileptic seizure activities detection and classification, IEEE International Conference on Acoustics, Speech and Signal Processing (ICASSP)", 2015.

14. G. Widman, T. Schreiber, B. Rehberg, A. Hoeft, and C. E. Elger, "Quantification of depth of anesthesia by nonlinear time series analysis of brain electrical activity”, Physical Review E, vol. 62, no. 4, 2000, pp. 4898-4903.

15. J. Gotman, "Automatic seizure detection: improvements and evaluation, Electroencephalography and Clinical Neurophysiology", vol. 76, no. 4, 1990, pp. 317-324.

16. L. Boubchir, S. Al-Maadeed, A. Bouridane, and A . Al Cherif,"Classification of EEG signals for detection of epileptic seizure activities based on LBP descriptor of time-frequency images", IEEE International Conference on Image Processing (ICIP), 2015

17. R. G. Andrzejak et al., "Indications of nonlinear deterministic and finite-dimensional structures in time series of brain electrical activity: dependence on recording region and brain state," Phys. Rev. E, vol. 64, no. 6, Nov. 2001, pp. 116-126.

18. Taran S, Bajaj V, Sharma D. Robust "Hermite decomposition algorithm for classifcation of sleep apnea EEG signals". Electron Lett. 2017;53(17):1182-4

19. S. Taran, and V. Bajaj, "Rhythm based identification of alcohol EEG signals," IET Scien., Measure.\&Tech.,DOI: 10.1049/iet-smt.2017.0232, 2017.

20. S. Taran, V. Bajaj, and S. Siuly, "An optimum allocation sampling based feature extraction scheme for distinguishing seizure and seizure-free EEG signals," Health infor. scien. and systems, vol. 5, no. 1, 2017, p.7.

21. LerchA."An introduction to audio content analysis: applications in signalprocessing and music informatics". Hoboken: Wiley; 2008.

22. Hassan AR, Haque MA. "Computer-aided obstructive sleep apnea screening from single-lead electrocardiogram using statistical and spectral features and bootstrap aggregating". Biocybern Biomed Eng. 2016;36(1):256-66.

23. Vapnik, V.:'The nature of statistical learning theory'(Springer-Verlag, New York,1995)

24. Dragomiretskiy K, Zosso D. "Variational mode decomposition". IEEE Trans Signal process. 2014;62(3):531-44.

25. Suykens, J.A., and Vandewalle, J.: "Least squares support vector machine classifiers. Neural processing letters", 9(3), 293-300, 1999

26. V. S. Vijith, J. E. Jacob, T. Iype, K. Gopakumar, and D. G. Yohannan, "Epileptic seizure detection using non linear analysis of EEG," in 2016 International Conference on Inventive Computation Technologies (ICICT), 2016, pp. 1-6.

27. D. Dilber and J. Kaur, "EEG based detection of epilepsy by a mixed design approach," in 2016 IEEE International Conference on Recent Trends in Electronics, Information \& Communication Technology (RTEICT), 2016, pp. 1425-1428.

28. M. M. o. Rashid and M. Ahmad, "Epileptic seizure classification using statistical features of EEG signal," in 2017 International Conference on Electrical, Computer and Communication Engineering (ECCE), 2017, pp.308-312. 